\title{
[ReView] Astrophysics of Dusty Stellar Winds from AgB Stars
}

\author{
KYUNG-WON SUH \\ Department of Astronomy and Space Science, Chungbuk National University, Cheongju 361-763, Korea \\ kwsuh@chungbuk.ac.kr
}

Received August 30, 2014; accepted October 14, 2014

\begin{abstract}
The main site of dust formation is believed to be the cool envelopes around AGB stars. Nearly all AGB stars can be identified as long-period variables (LPVs) with large amplitude pulsation. Shock waves produce by the strong pulsation and radiation pressure on newly formed dust grains drive dusty stellar winds with high mass-loss rates. IR observations of AGB stars identify various dust species in different physical conditions. Radio observations of gas phase materials are helpful to understand the overall properties of the stellar winds. In this paper, we review (i) classification of AGB stars; (ii) IR two-color diagrams of AGB stars; (iii) pulsation of AGB stars; (iv) dust around AGB stars including dusty stellar winds; (v) dust envelopes around AGB stars; (vi) mass-loss and evolution of AGB stars; and (vii) contribution of AGB dust to galactic environments. We discuss various observational evidences and their theoretical interpretations.
\end{abstract}

Key words: stars: AGB and post-AGB - circumstellar matter - infrared: stars - radiative transfer masers - dust: extinction

\section{INTRODUCTION}

An asymptotic giant branch (AGB) star is generally believed to be in the last evolutionary phases of a low mass star $\left(\mathrm{M} \leq 10 M_{\odot}\right)$. Most AGB stars can be identified as long-period variables (LPVs) with large amplitude pulsation. The strong pulsation produces shock waves which extend the outer layer of an AGB star for better condition of dust formation (e.g., Jones et al. 1981). The radiation pressure on newly formed dust grains may drive dusty stellar winds with high massloss rates of $10^{-8}-10^{-4} M_{\odot} / y r$ (e.g., Bowen 1988). As a result of the dusty stellar winds and evolution of the central stars during the AGB phase, the cool and slowly expanding (10-30 $\left.\mathrm{km} \mathrm{s}^{-1}\right)$ dust envelopes surrounding AGB stars are formed. AGB stars are believed to be major objects which are able to significantly affect the integrated spectral energy distributions (SEDs) of star clusters and galaxies (e.g., Cassarà et al. 2013).

The AGB phase is characterized by relatively rapid onset of greatly enhanced mass-loss, commonly referred to as the superwind phase. As AGB stars evolve, thermal pulses due to internal helium shell flashes have been hypothesized as a major cause of these episodes of greatly enhanced mass-loss (e.g., Suh \& Jones 1997). The mass-loss rate from an AGB star must increase by at least a factor of 10 for the duration of the superwind. We expect one thermal pulse every $10^{4}-10^{5}$ years that endures for a few hundred years (Iben \& Renzini 1983; Vassiliadis \& Wood 1993).

As stars evolve into the thermal pulsing AGB phase, the abundances of some elements in the stellar atmosphere may change by the episodic third dredge-up process after each thermal pulse. When AGB stars of in-

Corresponding AUthor: K.-W. Suh termediate mass range go through carbon dredge-up process and thus the $\mathrm{C} / \mathrm{O}$ ratio is larger than 1, O-rich dust grain formation ceases, and the stars may become visual carbon stars. After that phase, C-rich dust grains start forming (Groenewegen et al. 1995; Suh 2000).

The main site of dust formation is believed to be the cool envelopes around AGB stars. The envelopes are chemically fresh because of the strong binding force of $\mathrm{CO}$ molecules. O-rich dust grains (silicate; $\mathrm{Al}_{2} \mathrm{O}_{3}$; water ice) form in the O-rich envelopes and C-rich dust grains (amorphous carbon; $\mathrm{SiC}$ ) form in the C-rich envelopes. Then the dust grains may go through some physical and chemical changes after they are blown away from the AGB stars. Dust grains initially formed from molecules in AGB stars are generally believed to be in amorphous structure. The grains can be efficiently crystallized by annealing processes in high mass-loss rate AGB stars (e.g., Suh 2002). In interstellar medium, the crystalline grains can be transformed to be amorphous by ion bombardments (e.g., Kemper et al. 2004).

In this paper, we review general characteristics of dusty stellar winds from AGB stars by discussing various observational evidences and the theoretical interpretations. In the next section, we review classification of AGB stars: O-rich AGB stars, C-rich AGB stars, S stars and silicate carbon stars, a catalog of AGB stars, and the chemical evolution. We present IR two-color diagrams of AGB stars in Section 3. We discuss pulsation of AGB stars in Section 4. In Section 5, we review dust around AGB stars: dust formation in AGB stars, optical properties of dust grains, crystalline dust grains, and dust driven winds. Section 6 deals with dust envelopes around AGB stars: models for expanding dust shells, theoretical models for the 2CDs, and models for non-spherical multiple dust envelopes. In Section 7, we 


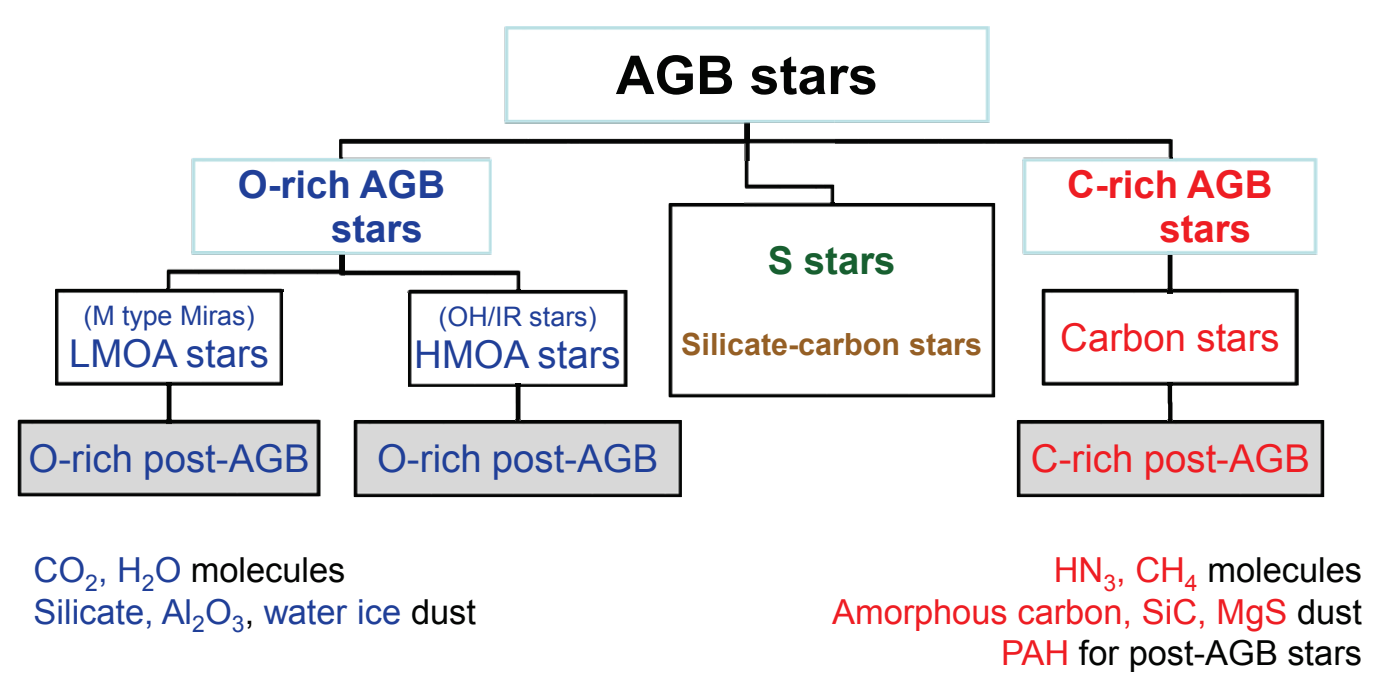

Figure 1. Classification of AGB stars based on chemistry of the central star and envelope.

review mass-loss and evolution of AGB stars: mass-loss from $\mathrm{OH} / \mathrm{IR}$ stars, evolution to post-AGB, and binary stars and dusty disks. We discuss contribution of AGB dust to galactic environments in Section 8. We conclude the paper with a summary in the last section.

\section{Classification of Agb Stars}

AGB stars are generally classified as O-rich (M-type) or C-rich (C-type) based on the chemistry of the photosphere and/or the outer envelope. Chan \& Kwok (1990) argued that an M-type star may become a carbon star when the star goes through $\mathrm{C}$ dredge-up processes and thus the abundance of $\mathrm{C}$ is larger than that of $\mathrm{O}$. S stars are generally regarded as intermediate between M-type and carbon stars in their properties. Figure 1 shows the conceptual diagram for the classification of AGB stars.

For a large sample of AGB stars, infrared observational data are available from the Infrared Astronomical Satellite (IRAS), Infrared Space Observatory (ISO), Midcourse Space Experiment (MSX), Two-Micron AllSky Survey (2MASS), Wide-field Infrared Survey Explorer (WISE) and AKARI. The IRAS point source catalog (PSC) version 2.1 contains useful photometric data in four bands $(12,25,50$ and $100 \mu \mathrm{m})$. The MSX (Egan et al. 2003) PSC provides useful photometric data in $8.28,12.13,14.65,21.34 \mu \mathrm{m}$ wavelength bands. The 2MASS (Cutri et al. 2003) PSC contains accurate positions and fluxes for about 470 million stars and other unresolved objects in $J(1.25 \mu \mathrm{m}), H(1.65 \mu \mathrm{m})$ and $K(2.17 \mu \mathrm{m})$ bands. The AKARI space telescope (Murakami et al. 2007) carried out an all sky survey with the infrared camera (IRC) and far infrared surveyor (FIS). We may use the AKARI PSC data in two bands (9 and $18 \mu \mathrm{m}$ ) obtained by the IRC and the brightsource catalogue (BSC) data in four bands (65, 90, 140 and $160 \mu \mathrm{m}$ ) obtained by the FIS.

The IRAS Low Resolution Spectrograph (LRS; $\lambda=$ $8-22 \mu \mathrm{m})$ data are useful to identify important dust features of AGB stars. The ISO Short Wavelength Spectrometer (SWS; $\lambda=2.4-45.4 \mu \mathrm{m}$ ) and the Long
Wavelength Spectrometer (LWS; $\lambda=43-197 \mu \mathrm{m}$ ) as well as the Spitzer Infrared Spectrograph (IRS; $\lambda=$ $5.2-38 \mu \mathrm{m})$ provided high resolution spectra for some AGB stars.

\subsection{O-Rich AGB Stars}

O-rich AGB stars (M-type Miras and OH/IR stars) typically show conspicuous $10 \mu \mathrm{m}$ and $18 \mu \mathrm{m}$ features in emission or absorption. They suggest the presence of amorphous silicate dust grains in the outer envelopes around them (e.g., Jones \& Merrill 1976). Further investigations showed that there are more dust species (e.g., $\mathrm{Al}_{2} \mathrm{O}_{3}$ and water-ice).

Low mass-loss rate O-rich AGB (LMOA) stars with thin dust envelopes show the $10 \mu \mathrm{m}$ and $18 \mu \mathrm{m}$ emission features of amorphous silicate. High mass-loss rate O-rich AGB (HMOA) stars with thick dust envelopes show the absorbing features at the same wavelengths. The high resolution ISO spectroscopic observations detected prominent emission from crystalline silicate in the infrared spectra of HMOA stars but not from the spectra of LMOA stars (Sylvester et al. 1999).

In IRAS LRS, O-rich AGB stars are classified into class of $\mathrm{E}(10 \mu \mathrm{m}$ in emission) and A $(10 \mu \mathrm{m}$ in absorption). Some of the class A or class E objects are young stellar objects or planetary nebulae. Kwok et al. (1997) used IRAS LRS to identify the class E $(10 \mu \mathrm{m}$ in emission) and the class A (10 $\mu \mathrm{m}$ in absorption) objects. Some of the class A or class E objects are young stellar objects or planetary nebulae.

Alumina $\left(\mathrm{Al}_{2} \mathrm{O}_{3}\right)$ dust is believed to be one of the main dust species in O-rich AGB stars (e.g., Jones et al. 2014). For modeling dust shells around O-rich AGB stars, Suh \& Kwon (2011) found that the dust opacity using $\mathrm{Al}_{2} \mathrm{O}_{3}$ as well as silicate can improve the fit of the dust model for the comparison with the IR observations. Water-ice features are found in SEDs of some high mass-loss rate OH/IR stars (e.g., Suh \& Kwon 2013a).

LMOA stars show many molecular lines at NIR 


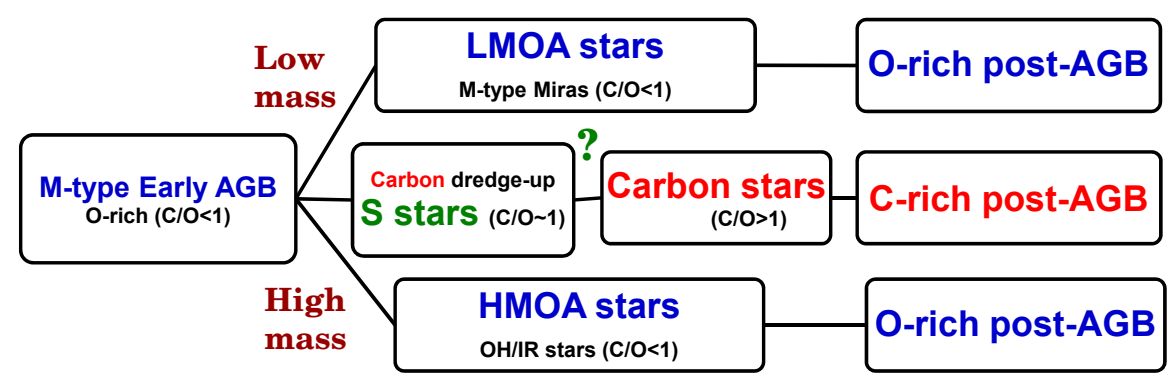

Figure 2. Possible evolutionary tracks for the classification of AGB stars.

Table 1

Maser sources in the sample of 3373 O-rich AGB stars

\begin{tabular}{llll}
\hline Maser & Reference & Detected & O-AGB \\
\hline $\mathrm{OH}$ & Suh \& Kwon (2011) & 3499 & 1533 \\
$\mathrm{SiO}$ & Kwon \& Suh (2012) & 1871 & 1627 \\
$\mathrm{H}_{2} \mathrm{O}$ & Kwon \& Suh (2012) & 2575 & 452 \\
no maser & Kwon \& Suh (2012) & & 610 \\
\hline Total & & & $3373^{1}$ \\
\hline
\end{tabular}

${ }^{1}$ Some stars are multiple maser sources.

bands. The most conspicuous lines are the water vapor absorption at 1.9 and $2.9 \mu \mathrm{m}$ and $\mathrm{CO}$ absorption at $2.3 \mu \mathrm{m}$ produced in the expanded atmosphere of the central stars. These absorption lines of $\mathrm{H}_{2} \mathrm{O}$ and $\mathrm{CO}$ make the $K$ band magnitude higher in the LMOA stars. Investigating the NIR molecular lines, Le Bertre et al. (2003) identified O-rich stars from the data obtained from IRTS.

$\mathrm{OH}, \mathrm{SiO}$, and $\mathrm{H}_{2} \mathrm{O}$ masers have been found to be associated with O-rich AGB stars (e.g., Kwon \& Suh 2012). Table 1 lists the maser sources in the sample of 3373 O-rich AGB stars.

\subsection{C-Rich AGB Stars}

The main components of dust in the envelopes around carbon stars are believed to be featureless amorphous carbon (AMC) grains and $\mathrm{SiC}$ grains producing the 11.3 $\mu \mathrm{m}$ emission feature (e.g., Suh 2000). The carbon stars with $\mathrm{SiC}$ grains belong to IRAS LRS class C. It was suggested that for carbon stars the $30 \mu \mathrm{m}$ band could be produced by small amount of MgS particles.

Unlike AGB stars, C-rich post-AGB stars typically show polycyclic aromatic hydrocarbon (PAH) dust features. This could be due to UV radiation from the hot central stars.

The carbon stars with thin dust envelopes show many molecular lines at NIR bands. The most conspicuous ones are the $\mathrm{HCN}$ and $\mathrm{C}_{2} \mathrm{H}_{2}$ absorption lines at $3.1 \mu \mathrm{m}$ produced in the expanded atmosphere of the central stars. Le Bertre et al. (2005) identified C-rich stars from the NIR data obtained with IRTS.
Table 2

A catalog of AGB stars

\begin{tabular}{lll}
\hline Class & Reference & Number \\
\hline O-rich & Kwon \& Suh (2012) & 3373 \\
C-rich & Suh \& Kwon (2011) & 1168 \\
S stars & Suh \& Kwon (2011) & 362 \\
Silicate carbon stars & Kwon \& Suh (2014) & $29\left(+27^{1}\right)$ \\
\hline
\end{tabular}

${ }^{1}$ Unconfirmed candidate objects.

\subsection{S Stars and Silicate Carbon Stars}

$\mathrm{S}$ stars are generally regarded as intermediate between M-type and carbon stars in their properties (e.g., Lloyd Evans \& Little-Marenin 1999). Only the S stars with Tc (also called intrinsic S stars) are believed to be actually in the AGB phase following the evolution sequence M-S-C (Iben \& Renzini 1983). However, Chan \& Kwok (1990) pointed out that the M-S-C evolutionary sequence is not a certain thing for all AGB stars.

Silicate carbon stars are carbon stars with silicate dust features (e.g., Kwon \& Suh 2014). They could be in the $\mathrm{M}-\mathrm{S}-\mathrm{C}$ chemical transition phase. At least some of these silicate carbon stars could be binary systems which had experienced a past event of becoming a carbon star from an O-rich AGB star.

\subsection{A Catalog of AGB Stars}

Suh \& Kwon (2011) presented a list of AGB stars for 3003 O-rich, 1168 C-rich, 362 S-type and 35 silicate carbon stars in our Galaxy which is a revised version of the catalog of AGB stars by Suh \& Kwon (2009a). For the catalog, they compiled previous works with verifying processes from the sources listed in the IRAS PSC. Kwon \& Suh (2012) presented a revised sample of 3373 O-rich AGB stars. Kwon \& Suh (2014) presented a revised sample of silicate carbon stars (29 confirmed objects and 27 unconfirmed objects). Therefore, the updated catalog lists 3373 O-rich, 1168 C-rich, 362 Stype and $29(+27)$ silicate carbon stars.

Table 2 summarizes the catalog of AGB stars. The catalog is open to the public through the web site http: //web.chungbuk.ac.kr/ kwsuh/agb.htm. 

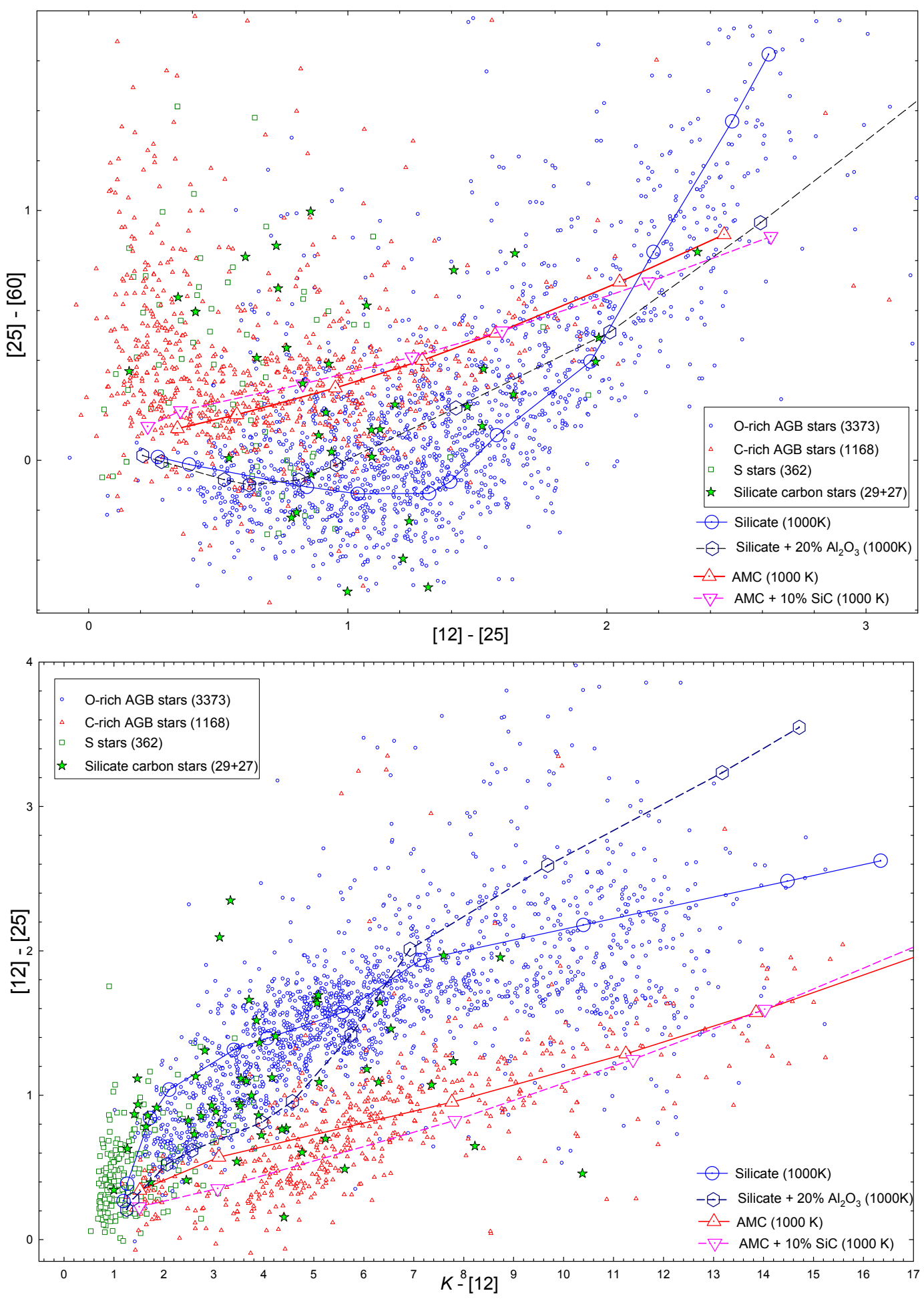

Figure 3. IR two-color diagrams for AGB stars.

\subsection{Chemical Evolution}

For different initial mass and metallicity, the chemical evolution would be very different (e.g., Iben 1981; Chan \& Kwok 1990). Assuming solar metallicity, Groenewegen (1995) and Blöcker et al. (2000) presented the following scenarios for different initial masses.
1) $\mathrm{M}<1.55 \mathrm{M}_{\odot}:$ They never become carbon stars because they experience only a small number of thermal pulses until the mass-loss by a stellar wind removes their outer envelope, which terminates their AGB evolution.

2) $1.55 \mathrm{M}_{\odot}<\mathrm{M}<2 \mathrm{M}_{\odot}:$ They become carbon stars after the star experiences its last thermal pulse 


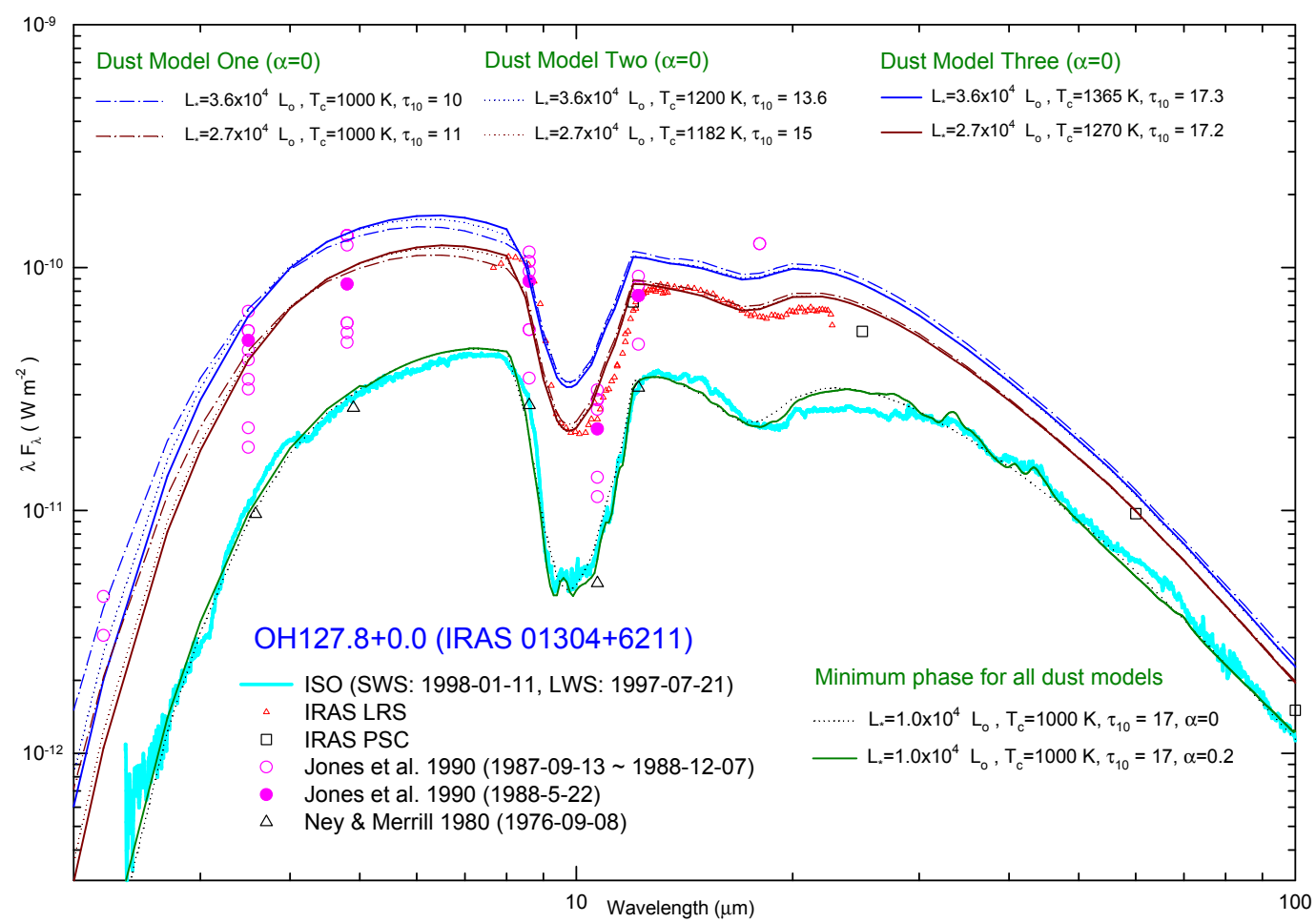

Figure 4. SEDs at different pulsation phases for $\mathrm{OH}$ 127.8+0.0. (from Suh 2004).

on the AGB. The stage of being an $\mathrm{S}$ star most likely is skipped.

3) $2 \mathrm{M}_{\odot}<\mathrm{M}<4 \mathrm{M}_{\odot}$ : They experience numerous thermal pulses, and they all become carbon stars at some point. During a few number of pulses, they pass through the S star phase .

4) $\mathrm{M}>4 \mathrm{M}_{\odot}$ : They become hot enough so that hot-bottom processing converts the carbon into ${ }^{14} \mathrm{~N}$ by means of the $\mathrm{CN}$ cycle. These stars do not become carbon stars. Though their surface element abundances undergo significant changes, they always remain M stars.

Figure 2 shows the conceptual diagram for the chemical evolution of AGB stars.

\section{IR Two-Color Diagrams of AGB Stars}

Only a relatively small number of AGB stars have complete or nearly complete SEDs. A large number of stars have infrared fluxes from IRAS, MSX, AKARI and NIR data. Although less useful than a full SED, the large number of observations with less extensive wavelength coverage can be used to form two-color diagrams (2CDs) that can be compared with theoretical model predictions.

IR colors are useful to characterize the dust envelopes around AGB stars. By using version 2.1 of the IRAS PSC, we obtain useful photometric data at three bands $(12,25$, and $60 \mu \mathrm{m})$ for AGB stars. We also use the 2MASS data at $K(2.17 \mu \mathrm{m})$ band. We cross-identify the 2MASS source by finding the nearest one in position.
The color index is defined as

$$
M_{\lambda 1}-M_{\lambda 2}=2.5 \log _{10} \frac{F_{\lambda 2} / Z M C_{\lambda 2}}{F_{\lambda 1} / Z M C_{\lambda 1}}
$$

where $Z M C_{\lambda i}$ means the zero magnitude calibration at given wavelength $(\lambda i)$ (see Suh \& Kwon 2011 for details). We use only those objects with good quality data at any wavelength.

Figure 3 shows the IR 2CD for the 3373 O-rich, 1168 C-rich, 362 S-type and $29(+27)$ silicate carbon stars (see Section 2.4 for the catalog of AGB stars). The small symbols are observational data and the lines with large symbols are theoretical model calculations for a range in dust-shell optical depth. We explain the theoretical models in Section 6.2. On these diagrams, the stars in the upper-right region have thick dust shells with large optical depths.

The upper panel of Figure 3 plots AGB stars in an IRAS 2CD using [25]-[60] versus [12]-[25]. The basic theoretical model tracks coincide roughly with the densely populated observed points. For O-rich stars, there is a change in the slope of the theoretical model line because the $10 \mu \mathrm{m}$ silicate feature changes from emission to absorption when the dust optical depth becomes larger. Carbon stars are distributed along a curve in the shape of a "C". A group of stars in the upper left region consists of optical carbon stars that show excessive flux at $60 \mu \mathrm{m}$, due to the remnant of an earlier phase when the stars were O-rich AGB stars (e.g., Chan \& Kwok 1990). A group of stars in the lower region, which extends to the right side, consists of infrared carbon stars. The infrared carbon stars on the 


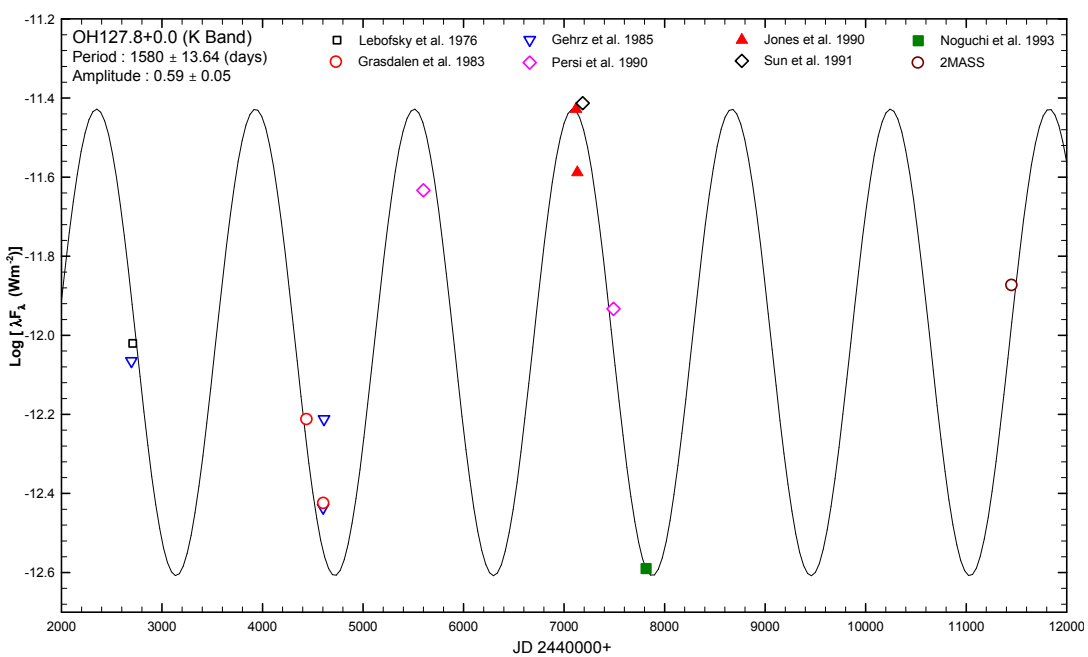

Figure 5. The $K$ band light curve of $\mathrm{OH} 127.8+0.0$. (from Suh \& Kwon 2009b).

right side have thick dust envelopes with large optical depths.

The lower panel of Figure 3 shows an IRAS and NIR $2 \mathrm{CD}$ using [12]-[25] versus $K-[12]$. Compared to the IRAS 2CD, the boundaries that separate O-rich, C-rich and $\mathrm{S}$ stars are clearer. We find that the basic theoretical model tracks roughly coincide with the densely populated observed points

Because the AKARI PSC measures the flux of the peak emission with a much smaller aperture $(9$ arcsec beam size) than IRAS PSC, the AKARI PSC position would be more useful for further identification.

\section{Pulsation of Agb Stars}

Nearly all AGB stars can be identified as LPVs with periods of 200 to 2000 days. The general catalog of variable stars (GCVS; Samus et al. 2011) contains the list of LPVs for different variable types. LPVs in AGB phase are classified according to the amplitude and regularity of the period into Miras (M), semi-regulars (SR), irregular (L), and RV Tauri type (RV) variables.

For many pulsating AGB stars, it has been known that the shapes of the SEDs as well as the overall luminosities vary depending on the phase of pulsation. The shapes of SEDs are affected by the properties of the dust shells as well as the central stars, depending on the phase of pulsation (e.g., Suh 2004).

For LMOA stars, the stronger stellar winds produce more dust grains and the dust shell optical depth increases at maximum phase of the bolometric luminosity. And at minimum phase of the luminosity, the weaker stellar winds produce fewer dust grains, and the dust shell optical depth decreases (Suh 2004). The innershell dust temperature is much lower than $1000 \mathrm{~K}$ and varies significantly during the pulsation phase $(\sim 400$ $\mathrm{K}$ at the minimum phase and $\sim 600 \mathrm{~K}$ at the maximum phase). However, the inner-shell dust temperature at the maximum phase is too low for the dust grains to be crystallized by annealing (see Section 5.3). This could be the reason why there are no crystalline silicate emis- sion features observed for LMOA stars.

There are phase lags in the optical-infrared light curves of LMOA stars (e.g., Smith et al. 2007). Most LMOA stars show phase lags in which the optical maximum precedes that in the near-infrared. Wittkowski (2007) found that the dust shell appears to be more compact with greater optical depth near visual minimum from mid-infrared interferometry of an LMOA star (S Ori) at four epochs. We need to consider the effect of phase lags for different wavelengths.

For HMOA stars, the deep silicate absorption features show significant variations depending on the pulsation phase, mainly due to changes in the properties of dust shells. The schemes of the SED changes are very different from those of LMOA stars. The environments for HMOA stars are suitable for the dust grains to be crystallized by annealing because of the hot dust temperature at the inner shell (see Section 5.3), especially at the maximum phase and with the slow velocity of the stellar winds.

Figure 4 shows the SEDs at different pulsation phases for an extreme HMOA star, OH 127.8+0.0 (Suh 2004 and references therein). Figure 5 shows The $K$ band light curve of $\mathrm{OH} 127.8+0.0$ (Suh \& Kwon 2009b and references therein). This star shows significant changes of bolometric luminosity over a pulsation cycle.

Suh \& Kwon (2009b), Kwon \& Suh (2010a), and Kwon \& Suh (2010b) investigated the $K, L$, and $M$ band light curves of $\mathrm{OH} / \mathrm{IR}$ stars, respectively. There is a general relationship between the period $(P)$ and amplitude $(A m p)$ for Mira-type LPVs. Figure 6 shows the period-amplitude (P-A) relation at $L$ band for $\mathrm{OH} / \mathrm{IR}$ stars obtained from Kwon \& Suh (2010a) and other investigations. A linear relationship from the data points is given by

$$
A m p=3.24 \log (P)-7.78 .
$$

The unused data points because of the high scatter from the general trend are marked by parenthesis. Table 3 lists the parameters for the P-A relation at three dif- 


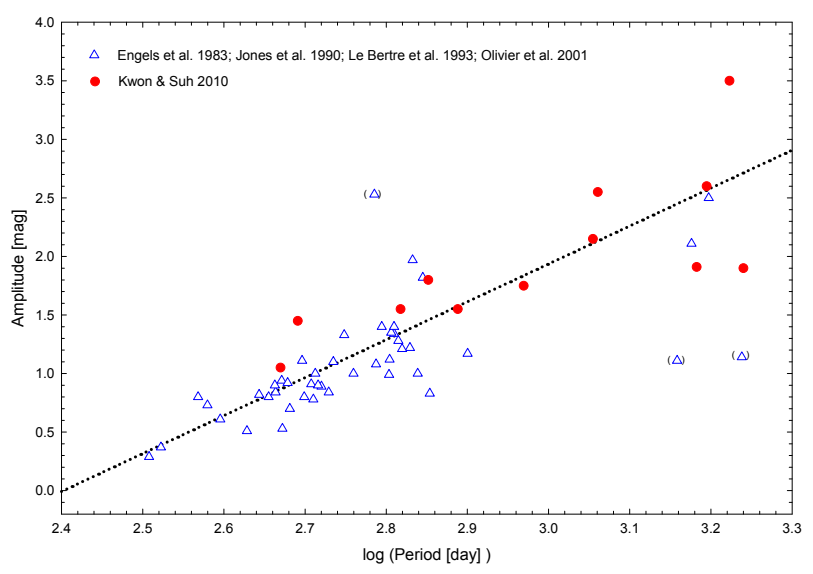

Figure 6. The period and amplitude relation for $\mathrm{OH} / \mathrm{IR}$ stars at $L$ band (from Kwon \& Suh 2010a).

ferent bands. When we compare the $\mathrm{P}$-A relation at different bands, the $M$ band shows a less steep slope and lower zero-point than those of $K$ and $L$ bands.

\section{Dust around AGB Stars}

For dust around AGB stars, there is a clear division between O-rich and C-rich dust grains because of the strong binding energy of the CO molecule. There are exceptions for transitional cases ( $\mathrm{S}$ stars and silicate carbon stars) and/or binary systems.

\subsection{Dust Formation in AGB Stars}

Studies of dust condensation around classical novae (Gehrz 1988) suggest that grains condense on time scales of several weeks, much shorter than the pulsation periods for AGB stars.

Although a complete theory for grain condensation is lacking, the work of Yamamoto \& Hasegawa (1977) provides a useful theoretical means of assessing the time scales involved in grain formation in the expanding circumstellar shells. For a typical AGB star (the stellar luminosity of $1 \times 10^{4} L_{\odot}$ and mass-loss rate of $\left.5 \times 10^{-5} M_{\odot} / y r\right)$, we obtain the grain condensation scale parameters from Kozasa, Hasegawa, \& Seki (1985). The saturation time for the grain seeds is computed to be about 1 day based on Deguchi (1980) for a condensation radius of $20 \mathrm{AU}$, corresponding to a dust condensation temperature of $1000 \mathrm{~K}$. The final grain radius is calculated to be about $0.1 \mu \mathrm{m}$ with these input parameter and is reached within one week (see Suh et al. 1990).

Both theoretical and empirical considerations agree that the grain formation time scale is much shorter than the pulsation periods of AGB stars. We expect that the dust size distribution is likely to be a Gaussian distribution rather than a power law which is more relevant to interstellar dust.

The dust-to-gas ratio $(\delta)$ is generally estimated to be 0.005 to 0.02 . Draine et al. (2007) derived the average dust-to-gas ratio in Milky way to be 0.01 and argued that the value tends to increase for a higher metallicity.
Table 3

$\mathrm{P}$-A parameters at different bands for $\mathrm{OH} / \mathrm{IR}$ stars

\begin{tabular}{llll}
\hline Band & Slope & Zero-point & Sample number \\
\hline$K$ & $3.17 \pm 0.57$ & $-6.89 \pm 1.69$ & 17 \\
$L$ & $3.24 \pm 0.22$ & $-7.78 \pm 0.62$ & 55 \\
$M$ & $2.81 \pm 0.22$ & $-6.57 \pm 0.62$ & 36 \\
\hline
\end{tabular}

\subsection{Optical Properties of Dust Grains}

Dust opacity is determined from the optical constants $\left(m_{\lambda}=n_{\lambda}+i k_{\lambda}\right)$, shape, and size of a dust grain.

The optical constants may be expressed by the complex index of refraction, $m=n+i k$, where $\varepsilon=m^{2}$. Derivation of the two functions of complex dielectric constants, $\varepsilon_{1}(\lambda)$ and $\varepsilon_{2}(\lambda)$, from the function $Q_{e x t}(\lambda)$ requires additional information. The supplementary physical constraint that the dielectric constants should satisfy is the Kramers-Kronig relation (Bohren \& Huffman 1983).

The optical constants of amorphous silicates derived by Suh (1999) resemble the experimental results for typical olivine silicate $\left(\mathrm{Mg}_{2 \mathrm{y}} \mathrm{Fe}_{2-2 \mathrm{y}} \mathrm{SiO}_{4} ; \mathrm{y}=0.4\right)$ obtained at room temperature by Dorschner et al. (1995). The laboratory measured optical data with largest content of $\mathrm{Fe}(\mathrm{x}=\mathrm{y}=0.4)$ fit best. When the amorphous silicates are crystallized to enstatite $\left(\mathrm{MgSiO}_{3}\right)$ or forsterite $\left(\mathrm{Mg}_{2} \mathrm{SiO}_{4}\right)$, there may be a process of Fe removal together with crystallization (Molster et al. 1999). The bulk density of amorphous silicate $\left(3.3 \mathrm{~g} \mathrm{~cm}^{-3}\right)$ is believed to be slightly higher than the density of crystalline silicate (Suh 2002).

Amorphous alumina $\left(\mathrm{Al}_{2} \mathrm{O}_{3}\right.$; Begemann et al. 1997) grains produce a single peak at $11.8 \mu \mathrm{m}$ and influences the shape of the SED at around $10 \mu \mathrm{m}$.

Figure 7 shows the opacity functions of major dust species in AGB stars. The opacity functions for O-rich dust species (silicate, alumina, and water-ice) as well as C-rich dust species (AMC and $\mathrm{SiC}$ ) are displayed. For silicate, the opacity functions for amorphous (cold and warm) grains and crystalline (forsterite and enstatite) grains are displayed. For the dust species for which the optical constants are available, the opacity functions are calculated for spherical dust grains using Mie theory (Bohren \& Huffman 1983). Table 4 summarizes the dust species.

\subsection{Crystalline Dust Grains}

Fabian et al. (2000) investigated the thermal evolution of amorphous silicates. They found that annealing at temperature $1000 \mathrm{~K}$ transformed amorphous silicate dust grains to crystalline ones in relatively short time scales. The crystallization took place within one day for the smoke materials and several days for the glass materials.

For HMOA stars, the dust formation temperature is believed to be about $1000 \mathrm{~K}$ (Suh 2004). If dust formation temperature is $1000 \mathrm{~K}$, the inner region of the outflowing envelope is hot (about 900-1000 K) during an extended period of time (several hundred days) 
Table 4

Dust species in AGB stars

\begin{tabular}{llllll}
\hline Acronym & Size $(\mu \mathrm{m})$ & Description & Density $(\mathrm{g} / \mathrm{cc})$ & Reference & Remark $^{a}$ \\
\hline SILc & 0.1 & amorphous cold silicate & 3.3 & Suh (1999) & optical constants \\
SILw & 0.1 & amorphous warm silicate & 3.3 & Suh (1999) & optical constants \\
FK & - & crystalline forsterite & 3.27 & Koike et al. (2003) & extinction curve \\
EK & - & crystalline enstatite & 3.27 & Chihara et al. (2002) & extinction curve \\
Alu & 0.1 & amorphous alumina & 3.2 & Begemann et al. (1996) & optical constants \\
ICE & 0.1 & crystalline water ice & 0.92 & Bertie et al. (1969) & optical constants \\
SWC & $0.1-0.2$ & SILc core, ICE mantle & 1.22 & - & optical constants \\
AMC & 0.1 & amorphous carbon & 2.0 & Suh (2000) & optical constants \\
SiC & 0.1 & $\alpha$-SiC & 3.26 & Pégourié (1988) & optical constants \\
\hline
\end{tabular}

${ }^{a}$ Type of the data in the reference.

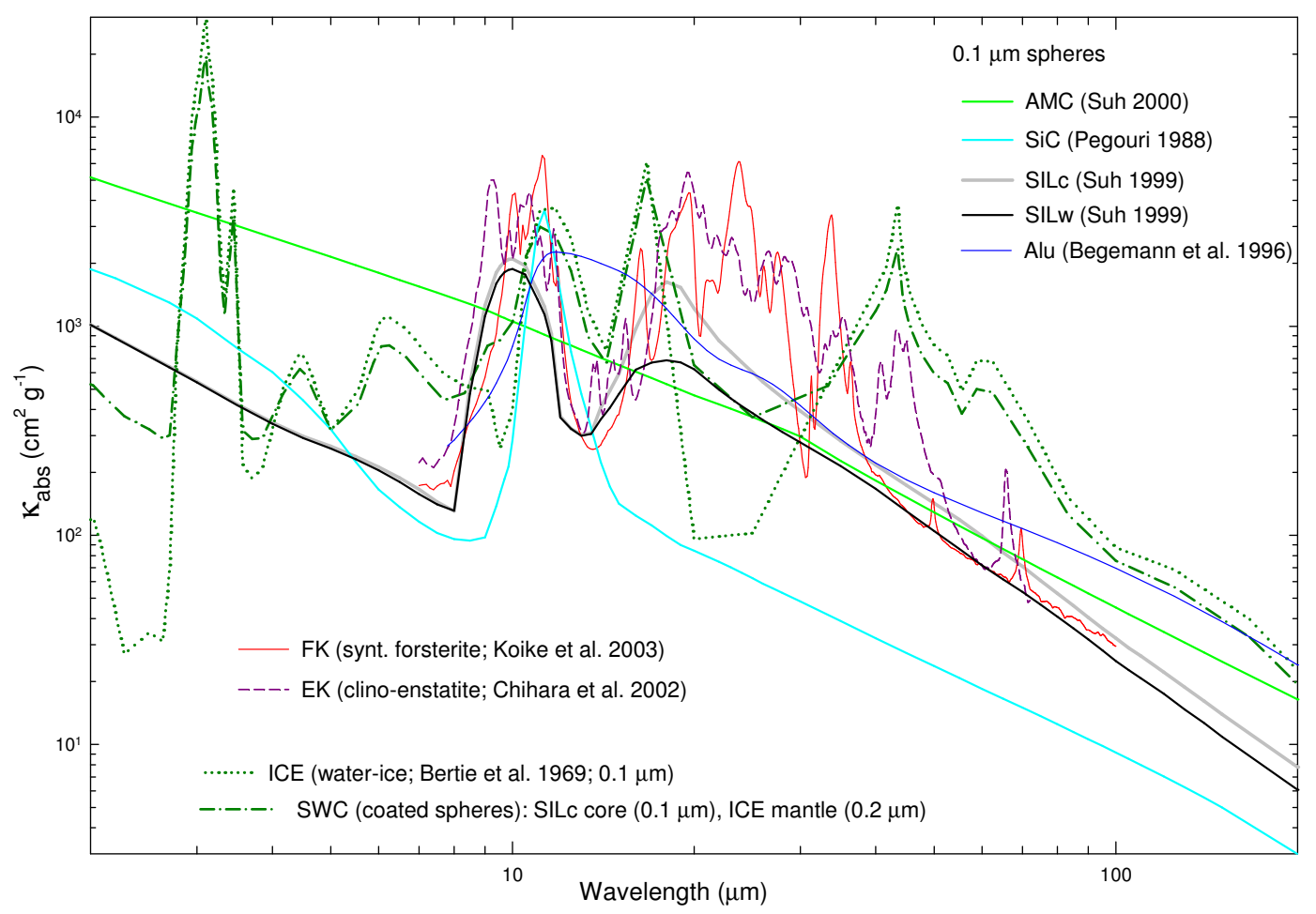

Figure 7. Opacity functions for dust species in AGB stars (see Table 4).

for a known crystallization process - annealing (e.g., Suh 2002; Suh 2004). Using the averaged single grain population model for mixed amorphous and crystalline silicates, Suh (2002) found that the dust shell models with about $10 \%$ to $20 \%$ of crystalline silicates produce the observed crystalline emission features for HMOA stars.

In the envelopes of LMOA stars for which the dust formation temperature is much lower than $1000 \mathrm{~K}$ (Suh 2004), the annealing would not be effective. This could be the reason why we find virtually no evidence of crystalline silicates for LMOA stars.

Dust grains initially formed from molecules in AGB stars are generally believed to be in amorphous structure. The grains can be efficiently crystallized by annealing processes in HMOA stars. In interstellar medium, the crystalline grains can be transformed to be amorphous by ion bombardments (e.g., Kemper et al. 2004).

\subsection{Dust Driven Winds}

The role of dust grains in driving mass-loss is well known. In absence of dust, the opacity in outer layers of AGB stars is very small. However, if one percent of the mass condenses into dust grains, the opacity can be increased by a thousand times, so that AGB stars can lose mass by radiation pressure on dust grains (e.g., Jones et al. 1981).

The outward radiation force per unit mass on spherical dust shell is

$$
f_{r a d}=\frac{\kappa_{p r}(r) L}{4 \pi c r^{2}}
$$




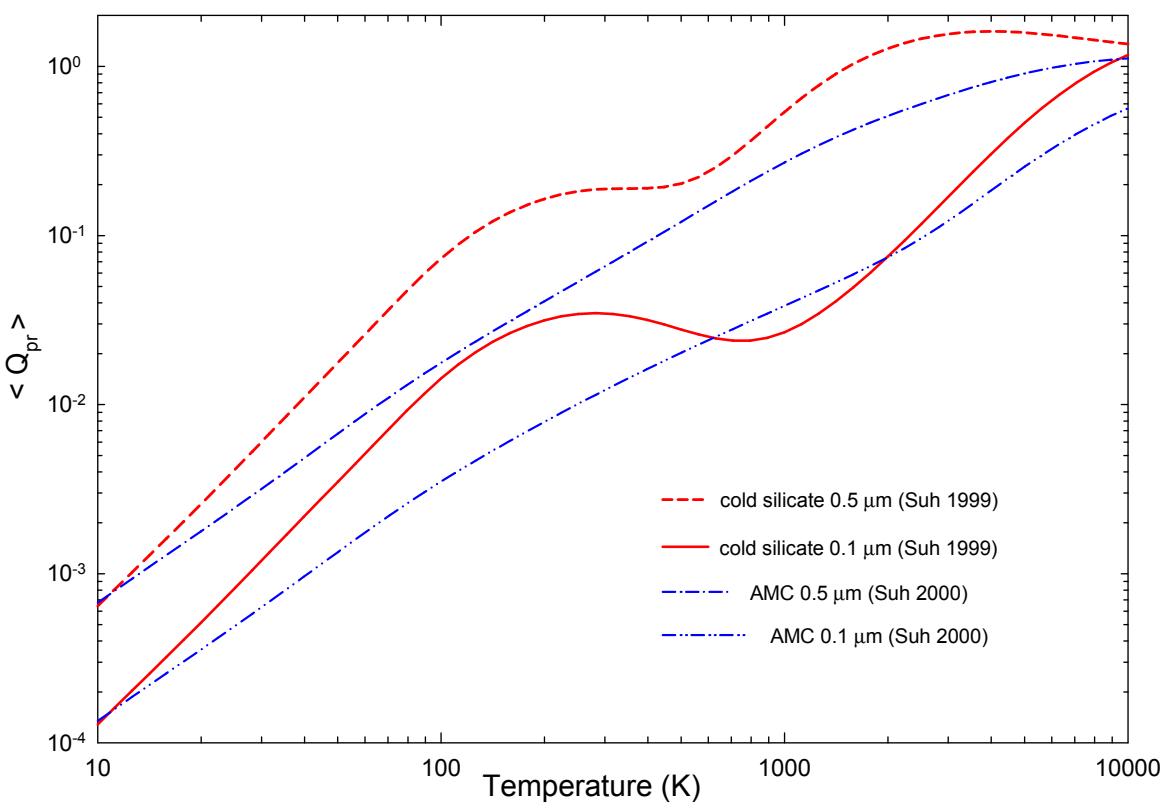

Figure 8. Planck mean values of the radiation pressure efficiency factors.

where $L$ is the luminosity of the central star, $c$ is speed of light, $r$ is the distance from the stellar center, and radiation pressure opacity $\kappa_{p r}\left(\mathrm{~cm}^{-1}\right)$ is given by

$$
\kappa_{p r}=\pi a^{2} N_{g}\left\langle Q_{p r}\right\rangle
$$

where $N_{g}$ is the dust grain number density, $\left\langle Q_{p r}\right\rangle$ is the Planck mean value of the radiation pressure efficiency factor $\left(Q_{p r}\right)$ which is given by

$$
Q_{p r}=Q_{e x t}-\langle\cos \theta\rangle Q_{s c a}
$$

where $Q_{\text {ext }}$ is the extinction efficiency factor, $Q_{s c a}$ is the scattering efficiency factor, and $\theta$ is the angle between the incident wave and the scattered wave.

The Planck mean value of the efficiency factor $Q(\lambda)$ is given by

$$
\begin{aligned}
\langle Q\rangle & \equiv 15\left(\frac{h c}{\pi k T}\right)^{4} \times \\
& \times \int_{0}^{\infty} Q(\lambda) \lambda^{-5}\left[\exp \left(\frac{h c}{\lambda k T}\right)-1\right]^{-1} d \lambda
\end{aligned}
$$

which is a function of the temperature. The Planck mean values of the absorption efficiency factors $\left(Q_{a b s}\right)$ are useful for estimating dust temperature. And the Planck mean values of the radiation pressure efficiency factors $\left(Q_{p r}\right)$ are useful for estimating the dynamical effects of dust grains which is important in studying stellar winds and mass-loss from AGB stars (e.g., Kozasa et al. 1984).

Figure 8 show the Planck mean values of the radiation pressure efficiency factors for silicate and AMC for different spherical grain radii. Once dust grains are formed in the envelopes around AGB stars, they can be efficiently accelerated faster than the escape velocity (e.g., Bowen 1988) by the radiation force (Equation (3)).

Radio observations of an AGB star may provide useful information to obtain the expansion velocity of the outer shell and the mass-loss rate of the gas phase materials. In Figure 9, we plot $V_{\text {exp }}$ obtained from the $\mathrm{CO}$ and $\mathrm{OH}$ maser observations versus the IR color for the sample $1533 \mathrm{OH} / \mathrm{IR}$ stars (see Suh \& Kwon 2013b). Large scatters in $V_{\text {exp }}$ could be due to pulsation. There is a general trend that $V_{\text {exp }}$ obtained from radio observations increases with the IR color for bluer objects (with smaller dust optical depths), while $V_{\text {exp }}$ levels off to a certain value for the redder objects. This trend of the gas expansion velocity (i.e., $V_{\text {exp }}$ obtained from radio observations) can be understood on the basis of the efficiency of dust-to-gas momentum transfer to the gas shell. When the mass-loss rate is small, any increase of the mass-loss rate causes a larger dust-gas coupling and so the momentum transfer increases. But when a critical value of the mass-loss rate is reached, further increase in the dust-to-gas momentum transfer for accelerating the gas phase materials is not effective (e.g., Kwok 1975; Stefen et al. 1997). See Section 7.1 for further discussion.

\section{Dust Envelopes around AgB Stars}

Dust grains in the outer envelopes of AGB stars absorb and scatter the stellar radiation and re-emit the radiation at longer wavelengths. For given characteristics of the central star and the dust envelope around it, the emergent SED can be found by analyzing the radiative transport processes. Comparison of these results with observations can be used to make adjustments and improvements in the model input parameters. To clarify 


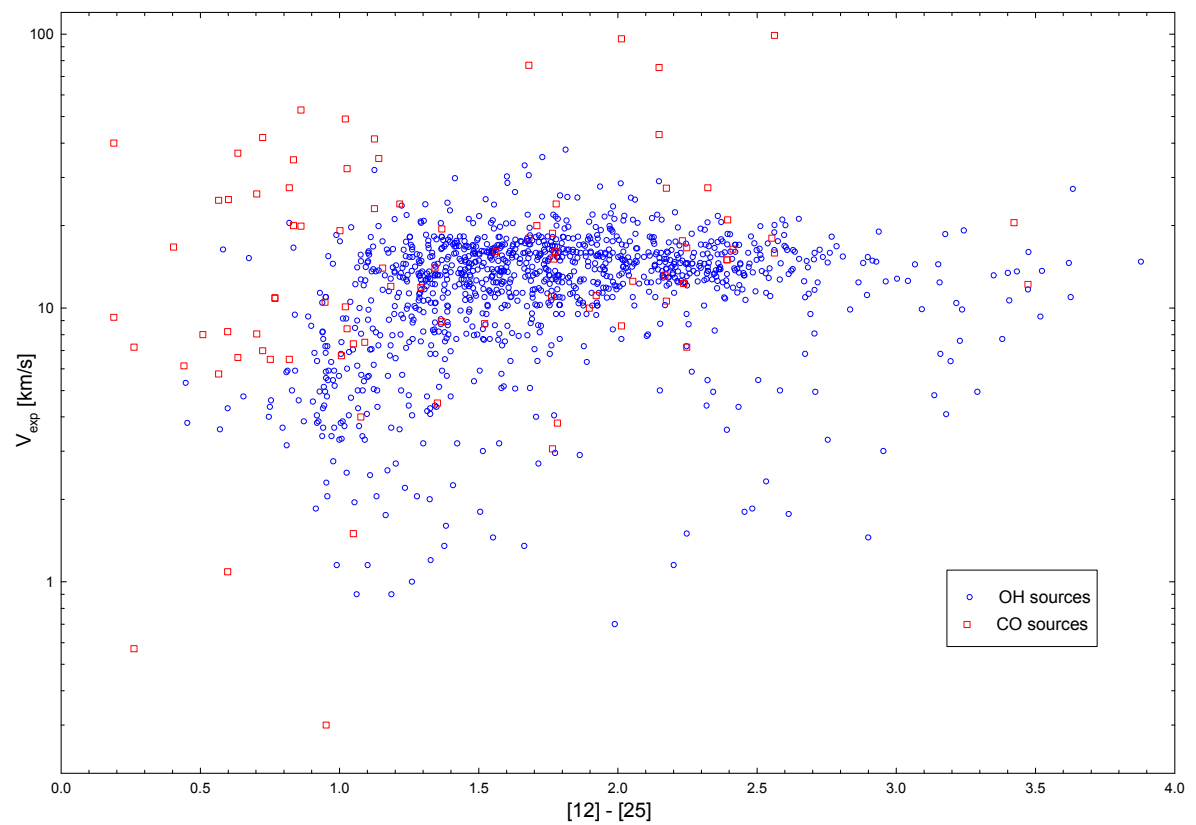

Figure 9. The $V_{\text {exp }}$ versus [12]-[25] diagram for $\mathrm{OH} / \mathrm{IR}$ stars.

the SED evolution of AGB stars, we need to use the opacity functions of the dust grains that are consistent with observations and physics.

\subsection{Models for Expanding Dust Shells}

Although AGB stars are pulsating, the overall continuous density distribution is believed to be maintained for a time scale larger than the pulsation period. Both simple radiation pressure models (e.g., Kwok 1975; Kozasa et al. 1984) or models that add pulsation and shocks (Bowen 1988; Suh et al. 1990) predict overall $\mathrm{r}^{-2}$ density laws. These models show that dust grains are accelerated relatively fast and approach and maintain terminal velocity within $3-5 R_{\text {in }}$. Dust formation and growth time-scales are very short (about a week) compared with other time-scales typical in AGB star winds (see Section 5.1). This burst-like grain formation implies a constant outflow velocity for most of the dust shell. The modest departures from constant velocity near $R_{i n}$ found by Suh et al. (1990) have only minor effects on the emergent spectrum.

For spherically symmetric dust shells, we assume that the dust density distribution is continuous $\left(\rho \propto r^{-2}\right)$ from the dust condensation radius $\left(R_{i n}\right)$ to the outer radius $\left(R_{\text {out }}\right)$ and the mass-loss rate $(\dot{M})$ and the envelope expansion velocity $\left(V_{\text {exp }}\right)$ are constant. Then the mass-loss rate is given by

$$
\dot{M}=4 \pi r^{2} \rho V_{\text {exp }} \simeq 4 \pi R_{i n}^{2} \rho_{o} V_{e x p},
$$

where $\rho_{o}$ is the material density at $R_{i n}$. The dust shell optical depth $\left(\tau_{\lambda}\right)$ is given by

$$
\tau_{\lambda}=\int \kappa_{\lambda} \rho_{d} d r \simeq \kappa_{\lambda} \rho_{d} R_{i n}
$$

where $\kappa_{\lambda}$ is the dust extinction opacity in $\mathrm{g}^{-1} \mathrm{~cm}^{2}$ and $\rho_{d}$ is the dust density at $R_{i n}$ which is given by

$$
\rho_{d}=\rho_{o} \delta
$$

where $\delta$ is the dust-to-gas ratio.

The inner shell dust temperature $\left(T_{c}\right.$; the dust condensation temperature) is the dust temperature at $R_{i n}$. The model SEDs are sensitively dependent on $T_{c}$. The temperature is not necessarily the same as the dust formation temperature, depending on the physical situations. For AGB stars, $T_{c}$ which fits the observed SEDs is calculated to be $500-1000 \mathrm{~K}$ (Suh 2004). The corresponding dust condensation radius (or inner-shell radius; $R_{\text {in }}$ ) is about 20 AU for HMOA stars and 50-68 AU for LMOA stars (see Suh 2004). At a typical expansion velocity $\left(V_{\text {exp }}\right)$ of $10-30 \mathrm{~km} \mathrm{~s}^{-1}$, it takes about 6 yrs to travel $1 R_{\text {in }}$ for HMOA stars. The outer radius of the dust shell is usually taken to be $10^{4}$ times the inner radius $\left(R_{i n}\right)$.

\subsection{Theoretical Models for the 2 CDs}

In this subsection, we explain the theoretical models presented in IR 2CDs (Figure 3; Section 3.1). We use the radiative transfer code DUSTY developed by Ivezić \& Elitzur (1997) for a spherically symmetric dust shell. We have performed the model calculations in the wavelength range 0.01 to $36000 \mu \mathrm{m}$. For all models, we assume the dust density distribution is continuous $\left(\rho \propto r^{-2}\right)$. The dust condensation temperature $\left(T_{c}\right)$ is assumed to be $1000 \mathrm{~K}$. The outer radius of the dust shell is always taken to be $10^{4}$ times the inner radius $\left(R_{c}\right)$. We choose $10 \mu \mathrm{m}$ as the fiducial wavelength that sets the scale of the dust optical depth $\left(\tau_{10}\right)$. For the central star, we assume that the luminosity is $10^{4} L_{\odot}$ for all models. 


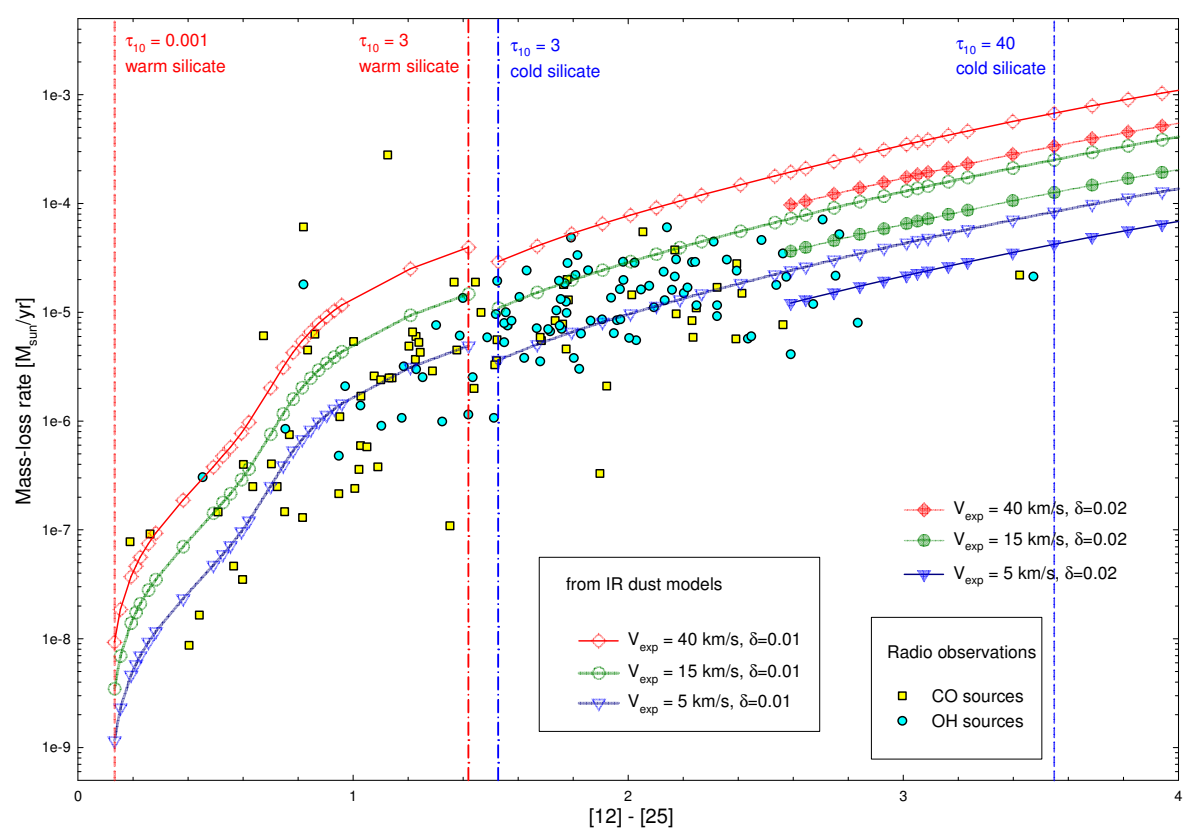

Figure 10. The mass-loss rate versus [12]-[25] diagram for OH/IR stars (from Suh \& Kwon 2013b).

For O-rich stars, we use a simple mixture of silicate and alumina ( $20 \%$ by mass) dust grains as well as pure silicate. For silicate, we use the optical constants of warm and cold silicate grains derived by Suh (1999). For alumina, we use the optical constants of amorphous alumina from Begemann et al. (1997). The radii of spherical dust grains are assumed to be $0.1 \mu \mathrm{m}$ uniformly. We perform the model calculations for eleven optical depths $\left(\tau_{10}=0.005,0.01,0.05,0.1,0.5,1,3,7\right.$, 15,30 and 40). We use the warm silicate dust grains for LMOA stars $\left(7\right.$ models with $\left.\tau_{10} \leq 3\right)$ and the cold grains for HMOA stars ( 4 models $\tau_{10}>3$ ). We assume that the stellar blackbody temperature is $2500 \mathrm{~K}$ for $\tau_{10} \leq 3$ and $2000 \mathrm{~K}$ for $\tau_{10}>3$. The IRAS flux at 12 $\mu \mathrm{m}$ is affected by addition of alumina dust (see Figure $3)$.

For C-rich stars, we use the optical constants of AMC grains derived by Suh (2000) and the optical constants of $\alpha \mathrm{SiC}$ grains by Pégourié (1988). The radii of spherical dust grains are assumed to be $0.1 \mu \mathrm{m}$ uniformly. We perform the model calculations for seven optical depths $\left(\tau_{10}=0.01,0.1,1,2,3,5\right.$ and 7$)$. We assume that the stellar blackbody temperature is $2300 \mathrm{~K}$ for $\tau_{10} \leq 0.1$ and $2000 \mathrm{~K}$ for $\tau_{10}>0.1$. We use a simple mixture of $\mathrm{AMC}$ and $\mathrm{SiC}(10 \%$ by mass) dust grains.

\subsection{Models for Non-Spherical Multiple Dust Envelopes}

Radiative transfer models for a single component and spherically symmetric dust shell (e.g., CSDUST3 code developed by Egan et al. 1988 and DUSTY code developed by Ivezić \& Elitzur 1997) have been very useful to investigate the general properties of dust envelopes around AGB stars. However, various dust species in the envelopes around AGB stars may have multiple components rather than a single component because of differ- ent formation temperatures. There are many observational evidences which implies that dust envelopes are not spherically symmetric.

Recently developed radiative transfer codes (e.g., RADMC-3D; HYPERION; SUNRISE) for investigating dust continuum radiative transfer processes based on the Monte Carlo simulation method (e.g., Bjorkman \& Wood 2001) can consider multiple 3-dimensional dust components to calculate the model spectra and images.

\section{Mass-Loss and Evolution of AGB Stars}

Stellar mass-loss is believed to be an essential property of cool, evolved AGB stars showing efficient dust condensation in the extended dynamic circumstellar envelope due to characteristic pulsation and thermal pulses (e.g., Bowen 1988). In the dynamical envelope, the efficient dust formation plays a key role for driving the wind by radiation pressure on the dust grains (e.g., Kwok 1975). This stellar wind generation mechanism is able to induce very large mass-loss rates $\left(10^{-8}-10^{-4} M_{\odot} / y r\right)$ in full consistency with observations and their theoretical interpretation.

\subsection{Mass-Loss from OH/IR Stars}

The $\mathrm{OH} / \mathrm{IR}$ stars are generally believed to be the last evolutionary phase of an O-rich AGB star emitting $\mathrm{OH}$ masers at $1612 \mathrm{MHz}$. OH/IR stars are characterized by Mira-type long-period variable stars with large amplitude pulsation and high mass-loss rates. The stellar wind of an $\mathrm{OH} / \mathrm{IR}$ star is driven by radiation pressure on dust grains and subsequent momentum transfer to the gas molecules via collisions (e.g., Kwok 1975). The mass-loss rate $(\dot{M}$; see Equation $(7))$ which can be derived from a dust shell model, is related to the dust shell optical depth $\left(\kappa_{\lambda} ; R_{i n} ; \rho_{d}\right)$ and $V_{\text {exp }}$. This can be 
compared with the ones obtained from radio observations.

Radio observations of an $\mathrm{OH} / \mathrm{IR}$ star may provide useful information to obtain the expansion velocity of the outer shell and the mass-loss rate. $\mathrm{OH}$ molecules produce a characteristic double-peak emission line from the shell of gas expanding away from the star. The difference in the velocities of the two peaks gives the expanding velocity of the gas. We need to know the brightness of the maser emission and the distance of the object as well as the expansion velocity to obtain the mass-loss rate (e.g., Zhang et al. 2010).

In Figure 10, we plot the diagrams of the mass-loss rates obtained from $\mathrm{OH}$ and $\mathrm{CO}$ observations versus the IR color ([12]-[25]) for OH/IR stars (from Suh \& Kwon 2013 b). The observed mass-loss rate increases with the IR color until it reaches about $10^{-5} M_{\odot} / y r$. After that, the mass-loss rate tends to maintain a certain level. In the region of low mass-loss rates $\left(\tau_{10} \leq 3\right)$, the theoretical mass-loss rates derived from the dust shell models generally agree with the radio observations. In the region of high mass-loss rate $\left(\tau_{10}>3\right)$, the theoretical mass-loss rates are obviously larger than the ones obtained from radio observations especially for higher dust optical depths. As we commented in Section 5.4, this could be because the photon momentum transfer to the gas shell is not effective for the physical condition of high mass-loss rates.

For high mass-loss rate $\mathrm{OH} / \mathrm{IR}$ stars, the outer gas shell radius which is measured by radio observations looks smaller than the outer dust shell radius which is measured by IR observations. For OH 26.5+0.6, Suh \& Kwon (2013a) could reproduce the observed image at $8.7 \mu \mathrm{m}$ obtained by Chesneau et al. (2005) using the dust model image of $R_{\text {out }}=3 \times 10^{4}$ AU. However in the $\mathrm{CO}(\mathrm{J}=1-0)$ image, the size of the object is much smaller (4700 AU; Fong et al. 2002). This may mean that the gas mass-loss is not as effective as the dust mass-loss for high mass-loss rate $\mathrm{OH} / \mathrm{IR}$ stars.

\subsection{Evolution to Post-AGB}

Unlike AGB stars, C-rich post-AGB stars typically show PAH dust feature (e.g., Kwok 2000). In the ISM, the aromatic features are emitted whenever interstellar PAH molecules or small carbonaceous grains containing PAHs are vibrationally excited by exposure to soft ultraviolet photons. In the circumstellar context, we expect PAHs to be abundant only in C-rich objects; and we expect a significant UV flux to be generally present only in objects that have evolved past the AGB.

For a major portion of planetary nebulae, the shape of the nebula is not spherically symmetric (e.g., Kwok 2000). The shapes of many of them look to be axisymmetric. They are often related with an equatorial disk (or torus) and bipolar flow. The physical origin for the non-spherical geometry could be stellar rotation, strong magnetic field, or binary evolution of the progenitor.

For a post-AGB star, the Red Rectangle (IRAS 06176-1036), which shows an equatorial disk, there are

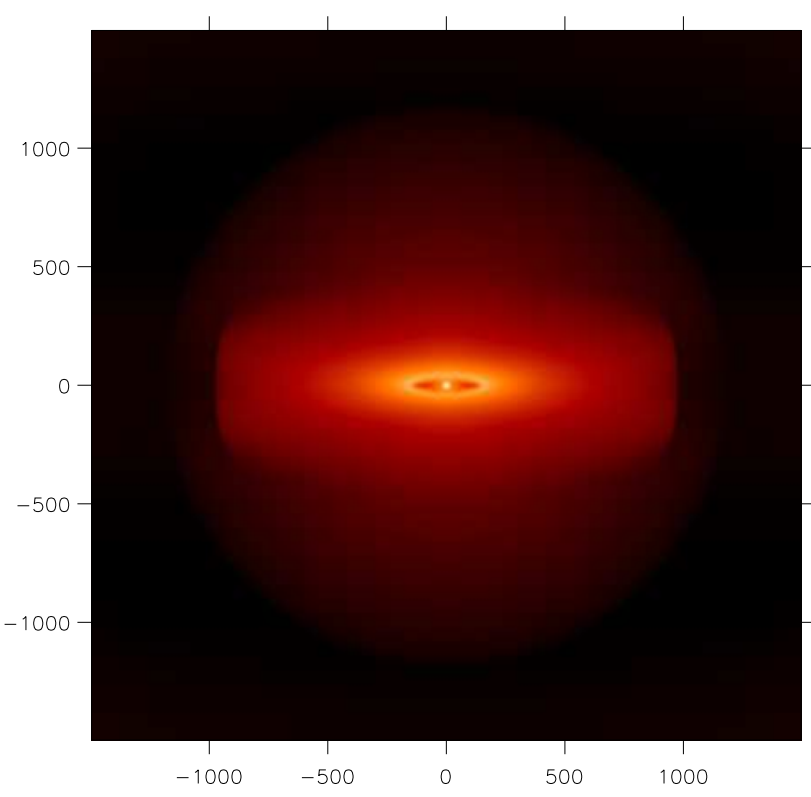

Figure 11. The model image for I09425 at $20 \mu \mathrm{m}$ (viewing angle $=75$ degrees). The 1000 AU scale of the figure corresponds to 560 mas at the distance of the object which is $1.8 \mathrm{kpc}$ (from K.-W. Suh 2014, in preparation).

strong evidences which support that the central object is a binary system (e.g., Men'shchikov et al. 2002). About 40 binary central stars of planetary nebulae are now known. As we will discuss in the next section, a binary system would be reasonably responsible for the equatorial disk.

\subsection{Binary Stars and Dusty Disks}

Silicate carbon stars are carbon stars which show circumstellar silicate dust features. We expect that most of them are likely to be binary systems rather than single stars (e.g., Kwon \& Suh 2014). Silicate carbon stars could have a low-luminosity companion and a relatively long-lived disk of silicate dust that is supposed to be formed when the primary was still an O-rich AGB star. There are some observational evidences for the presence of a disk in some silicate carbon stars. Ohnaka et al. (2008) made mid-IR interferometry observations toward BM Gem which suggested a circum-companion disk. For V778 Cyg, Szczerba et al. (2006) obtained high resolution $\mathrm{H}_{2} \mathrm{O}$ maser maps at $22 \mathrm{GHz}$ and they concluded that the object is composed of a C-rich star and a companion that stores an O-rich disk. Those observations are strong evidences for presence of an O-rich disk around a companion.

IRAS 09425-6040 (I09425) is a silicate carbon star with conspicuous crystalline silicate and water-ice features and excessive emission in the FIR and sub-mm wavelength regions (Molster et al. 2001). There is no direct observational evidence of binarity or presence of a disk for I09425 yet. Because circumstellar disks are believed to be suitable for growth of dust grains, we expect that the size of dust grains in a long-lived disk 
Table 5

Properties of dust grains in different regions

\begin{tabular}{llll}
\hline & AGB stars & Interstellar Dust & YSOs \\
\hline Chemistry & {$[\mathrm{O}]>[\mathrm{C}] \rightarrow$ O-rich dust $($ silicate, $\ldots)$} & Mixed & Mixed \\
& {$[\mathrm{O}]<[\mathrm{C}] \rightarrow$ C-rich dust $($ AMC,$\ldots)$} & & \\
Size & Larger size $(\sim 0.1 \mu \mathrm{m})$ & Smaller size $(\sim 0.01 \mu \mathrm{m})$ & Mixed size \\
Crystallization & LMOA: little & Generally weak & Protostars: $5-8 \%$ \\
& HMOA: high $(10-20 \%)$ & Diffuse: $<2.2 \%$ & Planetary systems: very high \\
Small graphite grains & Almost none & Abundant & Abundant \\
PAH & Only in post-AGB & Abundant & Abundant \\
\hline
\end{tabular}

would be very large (e.g., Gielen et al. 2008). Using a radiative transfer model with multiple 3 -dimensional dust envelopes, K.-W. Suh (2014, in preparation) finds that the observed SED requires very large dust grains in a circumbinay disk. Figure 11 shows the model images at $20 \mu \mathrm{m}$ for I09425.

For better understanding of origin and evolution of AGB and post-AGB stars, we need to consider the role of a binary system which is quite common.

\section{Contribution of AgB Dust to Galactic ENVIRONMENTS}

When the dust grains formed in AGB stars enter the interstellar medium (ISM), they get mixed up and go through some physical and chemical changes. There are similarities and differences between interstellar dust and dust grains in AGB stars. In interstellar medium, both O-rich and C-rich dust grains can exist together and the sizes of the dust grains are usually much smaller than found in AGB stars probably because of sputtering or grain-grain collision processes. In young stellar objects (YSOs), the dust grain size can be very large because of coagulation which dominates the grain growth in accretion disks.

Silicate grains can be efficiently crystallized by annealing processes in HMOA stars. In interstellar medium, the crystalline grains can be transformed to be amorphous by ion bombardments (e.g., Kemper et al. 2004). For C-rich dust grains, the crystallization of AMC to graphite or the significant chemical changes including hydrogenation could occur just after AGB phase or in interstellar medium. Table 5 summarizes the properties of dust grains in different regions of the Galaxy.

The contribution of dusty AGB stars to the SEDs of galaxies or stellar clusters is believed to be significant. The contribution to the IR radiation by the dusty shells of AGB stars is particularly relevant because AGB stars are luminous objects which are able to significantly affect the integrated SED of star clusters and galaxies (e.g., Piovan et al. 2003).

The SED of a galaxy reveals the extinction and reemission processes in the interstellar medium as well as the radiation from many types of stars. A theoretical population synthesis model is sensitively influenced by the property of the radiative processes and completeness of the spectral library of stars. The population synthesis model calculation combines several factors as the initial mass function, star formation rate, libraries of evolutionary tracks and model atmospheres which give an effect to the evolution of galaxies. Cassarà et al. (2013) investigated the role of dust in models of population synthesis. They used two libraries of about 600 AGB dust-enshrouded SEDs, one for O-rich M stars and one for carbon stars. These libraries of dusty AGB spectra are implemented into a large composite library of theoretical stellar spectra, to cover all regions of the Hertzsprung-Russell diagram crossed by the isochrones.

\section{SUMMARY}

AGB stars are generally classified as O-rich (M-type) or C-rich (C-type) based on the chemistry of the photosphere and/or the outer envelope. We have presented a catalog of AGB stars which lists 3373 O-rich, 1168 C-rich, 362 S-type and $29(+27)$ silicate carbon stars. IR 2CDs are presented for the AGB stars.

Most AGB stars are LPVs with large amplitude pulsation. Shock waves produced by the strong pulsation may efficiently extend the outer layer of an AGB star for better condition of dust formation. For many pulsating AGB stars, it has been known that the shapes of the SEDs vary as well as the overall luminosity depending on the phase of pulsation. The shapes of SEDs are affected by the properties of the dust shells as well as the central stars, depending on the phase of pulsation.

The main site of dust formation is believed to be the cool envelopes around AGB stars. For dust around AGB stars, there is a clear division between O-rich and C-rich dust grains because of the strong binding energy of the CO molecule. There are exceptions for transitional cases ( $\mathrm{S}$ stars and silicate carbon stars) and/or binary systems.

IR observations have identified various dust species and the physical interpretations are possible by reasonable theoretical models. Radio observations of gas phase materials are helpful to understand the overall properties of the stellar winds.

In the dynamical envelope of an AGB star, the efficient dust formation plays a key role for driving the wind by radiation pressure on the dust grains. This stellar wind generation mechanism is able to induce very large mass-loss rates. The stellar mass-loss plays a key role in the evolution of AGB and post-AGB stars as well as in the galactic environment. 
AGB stars are believed to be major objects which are able to significantly affect the integrated SEDs of star clusters and galaxies. For better understanding of origin and evolution of AGB and post-AGB stars, we need to consider the role of a binary system which is quite common. We may use the radiative transfer models which can consider multiple 3-dimensional dust components to calculate the model spectra and images.

\section{ACKNOWLEDGMEnTS}

This research was supported by Basic Science Research Program through the National Research Foundation of Korea (NRF) funded by the Ministry of Science, ICT \& Future Planning (NRF-2013R1A1A2057841).

\section{REFERENCES}

Begemann, B., Dorschner, J., Henning, T., et al. 1997, Aluminum Oxide and the Opacity of Oxygen-rich Circumstellar Dust in the 12-17 Micron Range, ApJ, 476, 199

Bertie, J. E. 1969, Absorptivity of Ice in the Range 4000-30 $\mathrm{cm}^{-1}$, The Journal of Chemical Physics, 50, 451

Bjorkman, J. E., \& Wood, K. 2001, Radiative Equilibrium and Temperature Correction in Monte Carlo Radiation Transfer, ApJ, 554, 615

Blöcker, T., Herwig, F., \& Driebe, T. 2000, AGB Evolution with Overshoot: Hot Bottom Burning and Dredge up, MmSAI, 71, 711

Bohren, C. F., \& Huffman, D. R. 1983, Absorption and Scattering of Light by Small Particles (New York: Wiley)

Bowen, G. H. 1988, Dynamical Modeling of Long-Period Variable Star Atmospheres, ApJ, 329, 299

Cassarà, L. P., Piovan, L., Weiss, A., Salaris, M., \& Chiosi, C. 2013, The Role of Dust in Models of Population Synthesis, MNRAS, 436, 2824

Chan, S. J., \& Kwok, S. 1990, Evolution of Infrared Carbon Stars, A\&A, 237, 354

Chesneau, O., Verhoelst, T., Lopez, B., et al. 2005, The Mid-IR Spatially Resolved Environment of OH 26.5+0.6 at Maximum Luminosity, A\&A, 435, 563

Chihara, H., Koike, C., Tsuchiyama, A., Tachibana, S., \& Sakamoto, D. 2002, Compositional Dependence of Infrared Absorption Spectra of Crystalline Silicates I : MgFe Pyroxenes, A\&A, 391, 267

Cutri, R. M., Skrutskie, M. F., Van Dyk, S., et al. 2003, The IRSA 2MASS All-sky Catalog of Point Sources. In: NASA/IPAC Infrared Science Archive

Deguchi, S. 1980, Grain Formation in Cool Stellar Envelopes, ApJ, 236, 567

Draine, B. T., Dale, D. A., Bendo, G., et al. 2007, Dust Masses, PAH Abundances, and Starlight Intensities in the SINGS Galaxy Sample, ApJ, 663, 866

Egan, M. P., Leung, C. M., \& Spagna, G. F. Jr. 1988, CSDUST3: a Radiation Transport Code for a Dusty Medium with 1-D Planar, Spherical or Cylindrical Geometry, Comput. Phys. Commun., 48, 271

Egan, M. P., Price, S. D., \& Kraemer, K. E. 2003, The Midcourse Space Experiment Point Source Catalog Version 2.3, BAAS, 203, 5708

Fabian, D., Jäger, C., Henning, T., et al. 2000, Steps toward Interstellar Silicate Mineralogy. V. Thermal Evolution of Amorphous Magnesium Silicates and Silica, A\&A, 364, 282

Fong, D., Justtanont, K., Meixner, M., \& Campbell, M.
T. 2002, Imaging the Circumstellar Envelope of $\mathrm{OH}$ 26.5+0.6, A\&A 396, 851

Gehrz, R. D. 1988, The Infrared Temporal Development of Classical Novae, ARA\&A, 26, 377

Gielen, C., Van Winckel, H., Min, M., et al. 2008, SPITZER Survey of Dust Grain Processing in Stable Discs around Binary Post-AGB Stars, A\&A, 490, 725

Groenewegen, M. A. T., van den Hoek, L. B., \& de Jong, T. 1995, The Evolution of Galactic Carbon Stars, A\&A, 293, 381

Iben, Jr. I. 1981, The Carbon Star Mystery - Why Do the Low Mass Ones Become Such, and Where Have All the High Mass Ones Gone, ApJ, 246, 278

Iben, I., \& Renzini, A. 1983, Asymptotic Giant Branch Evolution and Beyond, ARA\&A, 21, 271

Ivezić, A., \& Elitzur, M. 1997, Self-Similarity and Scaling Behaviour of Infrared Emission from Radiatively Heated Dust - I. Theory, MNRAS, 287, 799

Jones, O. C., Kemper, F., Srinivasan, S., et al. 2014, Modelling the Alumina Abundance of Oxygen-Rich Evolved Stars in the Large Magellanic Cloud, MNRAS, 440, 631

Jones, T. W., \& Merrill, K. M. 1976, Model Dust Envelopes around Late-Type Stars, ApJ, 209, 509

Jones, T. W., Ney, E. P., \& Stein, W. A. 1981, Pulsations, Grain Condensation, and Mass Loss in Long-Period Variable Stars, ApJ, 250, 324

Kemper, F., Vriend, W. J., \& Tielens, A. G. G. M. 2004, The Absence of Crystalline Silicates in the Diffuse Interstellar Medium, ApJ, 609, 826

Koike, C., Chihara, H., Tsuchiyama, A., et al. 2003, Compositional Dependence of Infrared Absorption Spectra of Crystalline Silicates II : Natural and Synthetic Olivine, A\&A, 399, 1101

Kozasa, T., Hasegawa, H., \& Seki, J. 1984, Grain Formation in the Expanding Gas Flow around Cool Luminous Stars, Ap\&SS, 98, 61

Kwok, S. 1975, Radiation Pressure on Grains as a Mechanism for Mass Loss in Red Giants, ApJ, 198, 583

Kwok, S., Volk, K., \& Bidelman, W. P. 1997, Classification and Identification of IRAS Sources with Low-Resolution Spectra, ApJS, 112, 557

Kwok, S. 2000, The Origin and Evolution of Planetary Nebulae (Cambridge: Cambridge University Press)

Kwon, Y.-J., \& Suh, K.-W. 2010a, Properties of the Variation of the Infrared Emission of OH/IR Stars II. The L Band Light Curves, JKAS, 43, 123

Kwon, Y.-J., \& Suh, K.-W. 2010b, Properties of the Variation of the Infrared Emission of OH/IR Stars III. The M Band Light Curves, JASS, 27, 279

Kwon, Y.-J., \& Suh, K.-W. 2012, Properties of OH, SiO, and $\mathrm{H}_{2} \mathrm{O}$ Maser Emission in O-Rich AGB Stars, JKAS, 45,139

Kwon, Y.-J., \& Suh, K.-W. 2014, A New Catalog of Silicate Carbon Stars, JKAS, 47, 123

Le Bertre, T., Tanaka, M., Yamamura, I., \& Murakami, H. 2003, Galactic Mass-Losing AGB Stars Probed with the IRTS, A\&A, 403, 943

Le Bertre, T., Tanaka, M., Yamamura, I., Murakami, H., \& MacConnell, D. J. 2005, Carbon Stars in the Infrared Telescope in Space Survey, PASP, 117, 199

Lloyd, E. T., \& Little-Marenin, I. R. 1999, Do S Stars Show Strong Silicate Dust Emission?, MNRAS, 304, 421

Men'shchikov, A. B., Schertl, D., Tuthill, P. G., Weigelt, G., \& Yungelson, L. R. 2002, Properties of the Close Binary and Circumbinary Torus of the Red Rectangle, A\&A, 393, 
867

Molster, F. J., Yamamura, I., Waters, L. B. F. M., et al. 1999, Low-Temperature Crystallization of Silicate Dust in Circumstellar Disks, Nature, 401, 563

Molster, F. J., Yamamura, I., Waters, L. B. F. M., et al. 2001, IRAS 09425-6040: A Carbon Star Surrounded by Highly Crystalline Silicate Dust, A\&A, 366, 923

Murakami, H., Baba, H., Barthel, P., et al. 2007, The Infrared Astronomical Mission AKARI, PASJ, 59, 369

Ohnaka, K., Izumiura, H., Leinert, C., et al. 2008, Asymmetric Silicate Dust Distribution toward the Silicate Carbon Star BM Geminorum, A\&A, 490, 173

Pégourié, B. 1988, Optical Properties of Alpha Silicon Carbide, A\&A, 194, 335

Piovan, L., Tantalo, R., \& Chiosi, C. 2003, Shells of Dust around AGB Stars: Effects on the Integrated Spectrum of Single Stellar Populations, A\&A, 408, 559

Samus, N. N., Durlevich, O. V., Kazarovets, E. V., et al. 2011, General Catalog of Variable Stars (GCVS database, Version 2011Jan), VizieR Online Data Catalogue, B/GCVS

Smith, B. J., Price, S. D., \& Moffett, A. J. 2007, Phase Lags in the Optical-Infrared Light Curves of AGB Stars, AJ, 131,612

Stefen, M., Szczerba, R., Men'schikov, A., et al. 1997, Hydrodynamical Models and Synthetic Spectra of Circumstellar Dust Shells around AGB Stars, A\&AS, 126, 39

Suh, K.-W., Jones, T. J., \& Bowen, G. H., 1990, A Pulsation Phase-dependent Dust Shell Model of OH 26.5+0.6, ApJ, 358,588

Suh, K.-W., \& Jones, T. J. 1997, Superwind Models for the Dust Shells around OH/IR Stars, ApJ, 479, 918

Suh, K.-W. 1999, Optical Properties of the Silicate Dust Grains in the Envelopes around Asymptotic Giant Branch Stars, MNRAS, 304, 389

Suh, K.-W. 2000, Optical Properties of the Carbon Dust Grains in the Envelopes around Asymptotic Giant Branch Stars, MNRAS, 315, 740

Suh, K.-W. 2002, Crystalline Silicates in the Envelopes and Discs around O-Rich AGB Stars, MNRAS, 332, 513
Suh, K.-W. 2004, Pulsation Phase-Dependent Dust Shell Models for Oxygen-Rich Asymptotic Giant Branch Stars, ApJ, 615, 485

Suh, K.-W., \& Kwon, Y.-J. 2009a, A Catalog of AGB Stars in IRAS PSC, JKAS, 42, 81

Suh, K.-W., \& Kwon, Y.-J. 2009b, Properties of the Variation of the Infrared Emission of $\mathrm{OH} / \mathrm{IR}$ Stars I. The $K$ Band Light Curves, JASS, 26, 279

Suh, K.-W., \& Kwon, Y.-J. 2011, Infrared Two-Colour Diagrams for AGB Stars Using AKARI, MSX, IRAS and Near-Infrared Data, MNRAS, 417, 3047

Suh, K.-W., \& Kwon, Y.-J. 2013a, Water Ice in High Massloss Rate OH/IR Stars, ApJ, 762, 113

Suh, K.-W., \& Kwon, Y.-J. 2013b, Mass-Loss Rates of OH/IR Stars, JKAS, 46, 235

Sylvester, R. J., Kemper, F., Barlow, M. J., et al. 1999, 2.4-197 $\mu \mathrm{m}$ Spectroscopy of OH/IR Stars: the IR Characteristics of Circumstellar Dust in O-Rich Environments, A\&A, 352, 587

Szczerba, R., Szymczak, M., Babkovskaia, N., et al. 2006, Oxygen-Rich Disk in the V778 Cygni System Resolved, A\&A, 452, 561

Vassiliadis, E., \& Wood, P. R. 1993, Evolution of Low- and Intermediate-Mass Stars to the End of the Asymptotic Giant Branch with Mass Loss, ApJ, 413, 641

Wittkowski, M., Boboltz, D. A., Ohnaka, K., Driebe, T., \& Scholz, M. 2007, The Mira Variable S Orionis: Relationships between the Photosphere, Molecular Layer, Dust Shell, and SiO Maser Shell at 4 Epochs, A\&A, 470, 191

Wright, E. L., Eisenhardt, P. R. M., Mainzer, A. K., et al. 2010, The Wide-Field Infrared Survey Explorer (WISE): Mission Description and Initial On-Orbit Performance, AJ, 140, 1868

Yamamoto, T., \& Hasegawa, H. 1977, Grain Formation through Nucleation Process in Astrophysical Environment, Progree of Theoretical Physics, 58, 816

Zhang, H.-J., Zhou, J.-J., Dong, G.-L., Esimbek, J., \& Mu, J.-M. 2010, The Properties of a Large Sample of OH/IR Stars, S Stars and C-Rich AGB Stars, ApSS, 330, 23 